\title{
Vigorous Endosteal Reaming across Fracture Site to Aid Union in Atypical Femur Fractures
}

\author{
Sanjay Agarwala, Mayank Vijayvargiya*, Prashant Pandey \\ P.D. Hiinduja National Hospital and MRC, Mumbai, India \\ Email: *maksy.doc37@gmail.com,drsa2011@gmail.com,prashant26292@gmail.com
}

How to cite this paper: Agarwala, S., Vijayvargiya, M. and Pandey, P. (2020) Paper Title. Open Journal of Orthopedics, 10, 412-418.

https://doi.org/10.4236/ojo.2020.1012039

Received: September 28, 2020

Accepted: December 26, 2020

Published: December 29, 2020

Copyright $\odot 2020$ by author(s) and Scientific Research Publishing Inc. This work is licensed under the Creative Commons Attribution International License (CC BY 4.0).

http://creativecommons.org/licenses/by/4.0/

\begin{abstract}
Background: Bisphosphonate abuse is associated with atypical femur fractures, which despite different fixation methods have increased non-union, delayed union, and re-operation rates. Therefore, there is a need for a surgical technique that may enhance union in such cases. Herein we are introducing a novel technique of sequential multiple vigorous reaming across the fracture site to introduce reaming debris/endosteal bone graft around the fracture site. Materials and Methods: We present a retrospective analysis of five prospectively treated patients with atypical diaphyseal femur fracture who presented to us from January 2015 to August 2019. All the patients were followed up at regular intervals and assessed both clinically and radiologically. Results: All 5 cases operated with our technique showed union at a mean follow-up of 16 weeks (12 - 20 weeks). There were no complications reported in our study at the last mean follow-up of 25 months (12 - 51 months). Conclusion: This technique of multiple vigorous reaming at and around the fracture site provides a new paradigm in the management of AFF. The autograft around the outer cortex promotes the formation of bridging callus and results in the early union.
\end{abstract}

\section{Keywords}

Atypical Femur Fracture, Vigorous Reaming, Bisphosphonates, Intramedullary Nail

\section{Introduction}

Long term bisphosphonate use is associated with the development of Atypical Femur Fractures (AFF). These are tensile stress fractures with defined radiographic features involving the femur from subtrochantericupto supracondylar flare. The term "atypical" was first coined in 1978 by Barcsa et al. in his descrip- 
tion of fatigue fractures [1], but Odvina et al. in 2005 were the first to publish a case report of bisphosphonate-related femoral fractures, suggesting a prominent pathogenic role of severe bone turnover suppression caused by these drugs [2]. These atypical femur fractures have simple transverse patterns, with unicortical beaking and hypertrophy of the diaphyseal lateral cortex and sustained after minimal to no trauma [3]. The surgical treatment of choice for AFFs is intramedullary nailing (IMN) or plating [3].

Despite following adequate principles of fracture fixation, these atypical diaphysealfractures are associated with increased non-union rates, delayed union rates and many times require multiple surgeries [4] [5]. Therefore, there is always a need for any change in surgical technique to enhance the rate of fracture union in such cases. The present article aimed to present 5 cases of atypical femur fracture induced by chronic bisphosphonate use and introducing a novel technique of sequential multiple vigorous reaming across the fracture site to introduce reaming debris/endosteal bone graft circumferentially around the fracture site.

\section{Materials and Methods}

We present a retrospective analysis of five prospectively treated patients with atypical diaphyseal femur fracture who presented to us from January 2015 to August 2019. All the patients were on Bisphosphonate therapy initiated and continued elsewhere and were discontinued on presentation. The mean age was 71 years (range 67 - 73 years) and all were females. There was no history of alcohol, smoking, or corticosteroid use. Patients diagnosed with complete atypical femoral fractures were included in this study. Those patients with active malignancy or bone metastasis, renal impairment were excluded from the study. Radiographs of the patients were studied by two independent senior orthopaedic surgeons to confirm atypical fracture characterized by the presence of cortical stress reaction, thickening, and flaring of the lateral cortex and transverse fracture pattern with minimal comminution at the fracture site [6].

Operative records of the cases subjected to surgery were screened for the mode of reduction, implant used, the surgical technique applied, and any intraoperative complications. Follow up was done on a regular basis until fracture union. Fixation was performed in the form of Intramedullary nailing in all the patients with special emphasis on vigorous reaming around the fracture site.

\section{Surgical Technique}

With the standard reaming technique, reaming is started with the smallest size flexible reamer over the guidewire and is repeatedly reamed to and fro across the fracture site. This step is then repeated with each increasing size reamers until an adequate cortical purchase is obtained. This vigorous sequential multiple to and fro reaming at the fracture site allows bone dust pushed through the fracture gap to distribute in and around the fracture site to form an envelope which is visible 
on the image intensifier. Reamer used was flexible, power-operated, low velocity, and high torque. Figure 1 and Figure 2 shows illustrative case examples of our technique.

All the patients were followed up at 2 weeks, 6 weeks, 3 months, 6 months, and 6 monthly thereafter till fracture union. Patients were assessed both clinically (assessment of wound, tenderness at fracture site, and ability to bear weight) and radiologically (anteroposterior and lateral radiographs of the femur were taken and assessed for the radiological union at fracture site). Fracture was termed as united when bridging callus formation was seen in AP and lateral views along with no fracture gap.
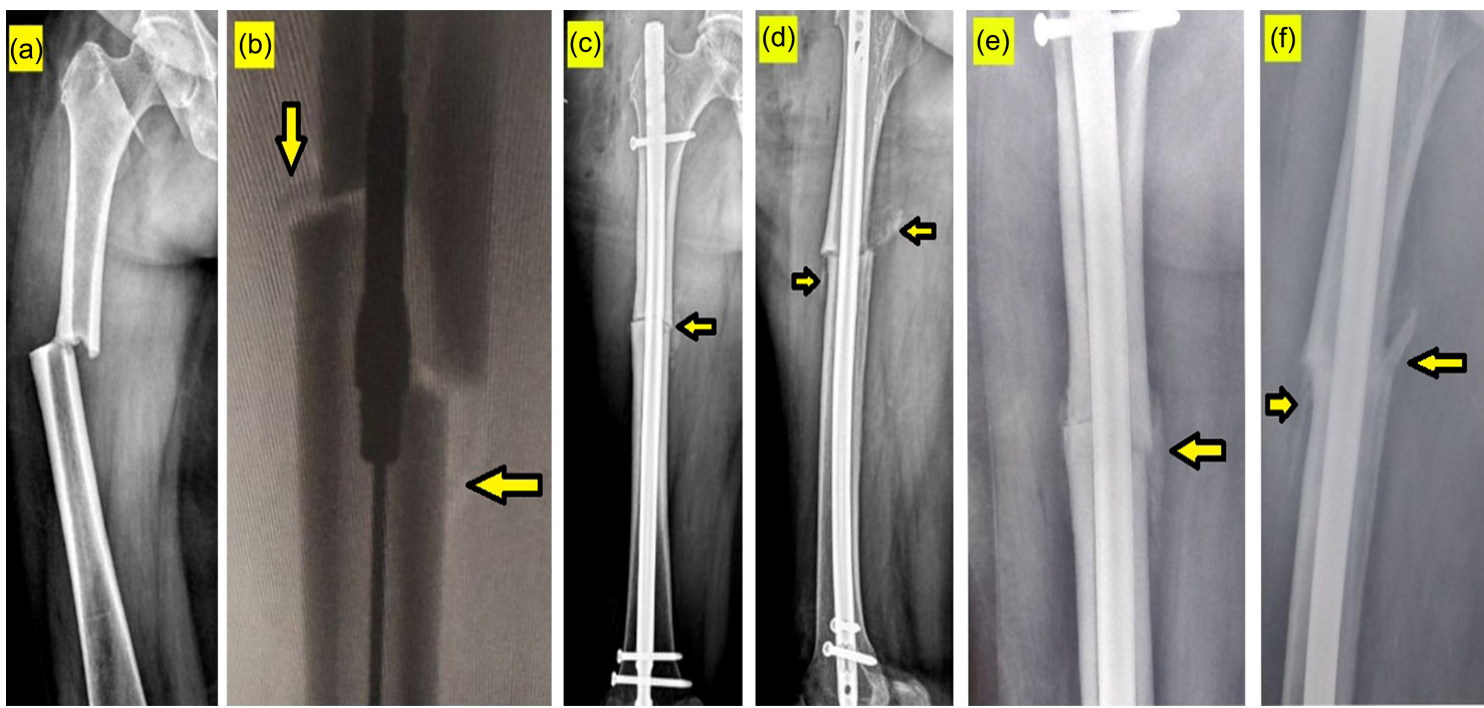

Figure 1. (a) Pre-operative radiograph showing Atypical Diaphyseal femur fracture in a 73 year old patient; (b) Intra-operative intensifier image showing reamer at the fracture site leading to the flocculus/bone dust (arrow) being pushed from the fracture gap and getting distributed around the fracture site; (c), (d) Immediate post-operative radiograph showing bone dust around the fracture area (arrow); (e), (f) Follow-up radiograph at 12 weeks showing complete union and incorporation of bone dust as a bridging callus across the fracture site (arrow).
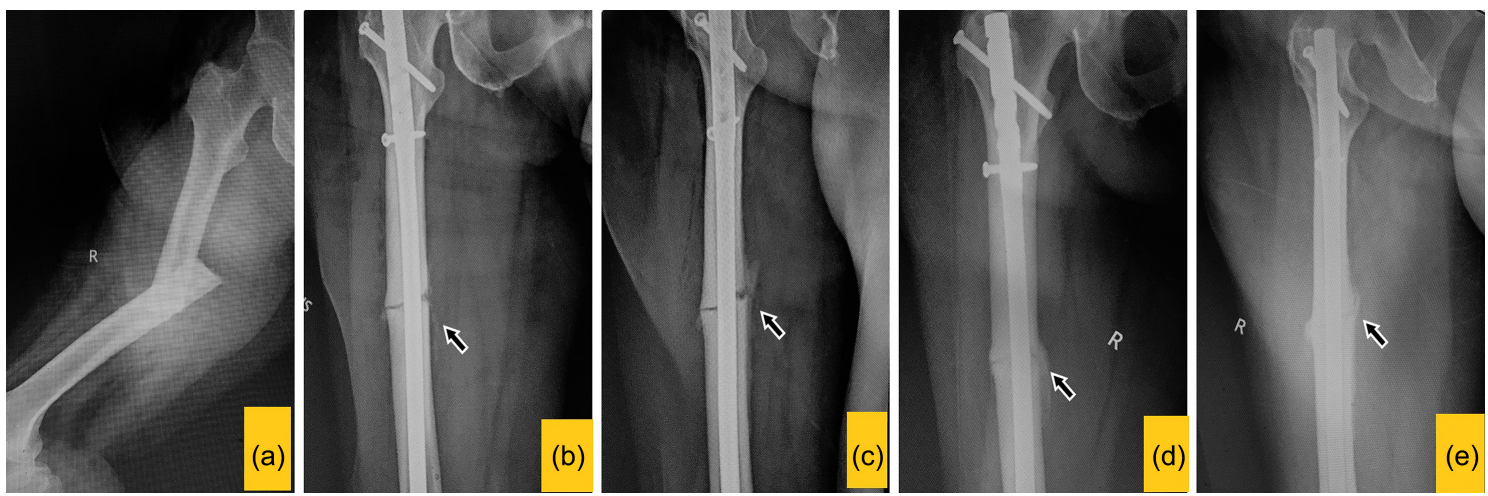

Figure 2. (a) Pre-operative lateral radiograph showing Atypical Diaphyseal femur fracture in a 73 years old patient; (b), (c) Immediate post-operative radiograph (anteroposterior and lateral) showing fixation using an intramedullary nail with bone dust around the fracture area (arrow); (d), (e) Follow-up radiograph (anteroposterior and lateral) at 18 weeks showing complete union and incorporation of bone dust as a bridging callus across the fracture site (arrow). 


\section{Results}

A total of 5 patients were available for the analysis. Three of the fractures were on the right, two on the left side (Table 1). All the patients had sustained the fracture while walking without any history of significant trauma. None of the patients had significant risk factors before initiation of Bisphosphonate treatment. Configurations of fractures are shown in Table 1. All patients except for one presented immediately after a displaced fracture. One patient was already treated for the displaced fracture with external fixation before being referred to our center.

All the five cases showed union at a mean follow-up of 16 weeks (12 - 20 weeks). All the patients treated by us were kept strict non-weight bearing with crutch support till radiographic evidence of callus formation was seen. Followed by partial weight-bearing till union was achieved on radiographs. There are no complications reported in our cases until the last mean follow-up of 25 months (12 - 51 months).

\section{Discussion}

Atypical femur fractures are known to have a delayed union with a mean time to union of 7.3 months, this is longer than the union time seen in typical femoral fractures which heals between 3 - 6 months [4] [5]. In our study with vigorous reaming around the fracture site, we have observed that all the fractures have united with a mean time of 16 weeks (12 - 20 weeks).

Despite the various modes of fixation, these fractures are known to have higher non-union, delayed union, and reoperation rates. Studies have shown that intramedullary nailing is biomechanically better than plate fixation in atypical femur fracture, due to its load-sharing properties and allowing less bending movements at the fracture site. In a retrospective analysis of 33 patients with atypical femur fractures by Teo et al., the authors have reported that the mean time to union with plate fixation was 10.9 months and with intramedullary nailing was 7.7 months [7].

Table 1. Demographics and treatment details of the patient included in this study.

\begin{tabular}{cccccccc}
\hline Age & Bisphosphonate & $\begin{array}{c}\text { Duration of } \\
\text { therapy (years) }\end{array}$ & Affected side & Treatment & Time to union & Outcome \\
\hline 70 & Alendronate & 5 & Left & I.M Nailing with Vigorous reaming & 14 weeks & Healed \\
73 & Alendronate & 7 & Right & I.M Nailing with Vigorous reaming & 12 weeks & Healed & Healed \\
72 & Zolendronic acid & 5 & Right & I.M Nailing with Vigorous reaming & 20 weeks & Healed \\
73 & Alendronate & 6 & Left & I.M Nailing with Vigorous reaming & 16 weeks & Healed \\
\hline
\end{tabular}


Current literature shows conflicting results for intramedullary nailing in atypical femur fractures. Egol et al. have shown a union rate of $98 \%$ whereas Weil et al. have shown that $46 \%$ of the fractures treated with intramedullary nailing required revision surgery [8] [9]. Therefore, with such a contrasting difference in the union rate with the use of intramedullary nailing, it is clear that just nailing is not the solution for these atypical femur fractures.

The literature suggests that surgical treatment of AFF is more complex than that of typical femoral fractures, healing time is prolonged and reduction and surgical technique is more demanding, leaving little room for error [10]. Therefore, we have an improvised reaming technique with a specific focus on sequential multiple vigorous reaming. The bone dust/floccules deposited not only provide a scaffold (osteoconduction) but also have osteogenic and osteoinductive properties [11] [12]. Further, this technique also introduces microfractures at the fracture site, which promotes fracture healing.

We have used this technique in 5 cases of bisphosphonates induced atypical femur shaft fractures which showed union at a mean follow-up of 16 weeks (12 20). The autograft around the outer cortex promotes the formation of bridging callus and results in the early union. This technique is easy to use with no added cost and special requirement.

Studies have shown shorter time to union, lower delayed union rate with reamed intramedullary nailing as compared to unreamed nailing [11]. Although various animal studies have proposed that reaming intramedullary nailing may have dramatic acute effects on the diaphyseal blood flow. They have proposed that reaming removes the marrow contents, endosteum, inner cortical bone and destroys the medullary blood vessels resulting in an initial decreased blood flow and may lead to cortical bone necrosis in the inner half of the cortex [13] [14] [15]. However, no clinical studies have supported these findings and various authors have reported no adverse effects of reamed intramedullary reaming [11] [16].

\section{Limitation}

We have presented a small case series of 5 patients but considering the incidence of AFF to be very rare and the fact that we have focused on a single mode of surgical treatment compared to other studies focusing on multiple modalities of management, hence we consider this study to provide a pragmatic solution to the management of atypical femur fractures.

\section{Conclusion}

All 5 cases of atypical femur fracture included in our study showed union at a mean follow-up of 16 weeks (12 - 20 weeks). Thus, this technique of multiple vigorous reaming at and around the fracture site provides a new paradigm in the surgical technique for the management of AFF, which otherwise has high non-union, delayed union, and re-operation rates. The autograft around the 
outer cortex promotes the formation of bridging callus and results in the early union.

\section{Conflicts of Interest}

The authors declare no conflicts of interest regarding the publication of this paper.

\section{References}

[1] Barcsa, C., Endes, J. and Szappanos, L. (1978) Atypical Fatigue Fractures. Beiträge zur Orthopädie und Traumatologie, 25, 578-582.

[2] Odvina, C.V., Zerwekh, J.E., Rao, D.S., Maalouf, N., Gottschalk, F.A. and Pak, C.Y.C. (2005) Severely Suppressed Bone Turnover: A Potential Complication of Alendronate Therapy. The Journal of Clinical Endocrinology \& Metabolism, 90, 1294-1301. https://doi.org/10.1210/jc.2004-0952

[3] Spinelli, M.S., Marini, E., Daolio, P.A. and Piccioli, A. (2019) Atypical Diaphyseal Femoral Fractures: Considerations on Surgical Technique. Injury, 50, S65-S69. https://doi.org/10.1016/j.injury.2019.01.048

[4] Koh, A., Guerado, E. and Giannoudis, P.V. (2017) Atypical Femoral Fractures Related to Bisphosphonate Treatment: Issues and Controversies Related to Their Surgical Management. The Bone \& Joint Journal, 99, 295-302. https://doi.org/10.1302/0301-620X.99B3.BJI-2016-0276.R2

[5] Agarwala, S., Agashe, V.M., Shetty, V., et al. (2016) Caveats of Bisphosphonate Abuse. Indian Journal of Orthopaedics, 50, 434-439. https://doi.org/10.4103/0019-5413.185612

[6] Koh, J.S., Goh, S.K., Png, M.A., Ng, A.C. and Howe, T.S. (2011) Distribution of Atypical Fractures and Cortical Stress Lesions in the Femur: Implications on Pathophysiology. Singapore Medical Journal, 52, 77-80.

[7] Teo, B.J., Koh, J.S., Goh, S.K., et al. (2014) Post-Operative Outcomes of Atypical Femoral Subtrochanteric Fracture in Patients on Bisphosphonate Therapy. The Bone \& Joint Journal, 96, 658-664. https://doi.org/10.1302/0301-620X.96B5.32887

[8] Egol, K.A., Park, J.H., Rosenberg, Z.S. and Peck VmTejwani, N.C. (2014) Healing Delayed But Generally Reliable after Bisphosphonate-Associated Complete Femur Fractures Treated with IM Nails. Clinical Orthopaedics and Related Research, 472, 2728-2734. https://doi.org/10.1007/s11999-013-2963-1

[9] Weil, Y.A., Rivkin, G., Safran, O., Liebergall, M. and Foldes, A.J. (2011) The Outcome of Surgically Treated Femur Fractures Associated with Long-Term Bisphosphonate Use. The Journal of Trauma, 71, 186-190.

https://doi.org/10.1097/TA.0b013e31821957e3

[10] Prasarn, M.L., Ahn, J., Helfet, D.L., Lane, J.M. and Lorich, D.G. (2012) Bisphosphonate-Associated Femur Fractures Have High Complication Rates with Operative Fixation. Clinical Orthopaedics and Related Research, 470, 2295-2301. https://doi.org/10.1007/s11999-012-2412-6

[11] Li, A.B., Zhang, W.J. and Guo, W.J. (2016) Reamed versus Unreamed Intramedullary Nailing for the Treatment of Femoral Fractures: A Meta-Analysis of Prospective Randomized Controlled Trials. Medicine (Baltimore), 95, e4248. https://doi.org/10.1097/MD.0000000000004248

[12] Porter, R.M., Liu, F., Pilapil, C., et al. (2009) Osteogenic Potential of Reamer Irrigator Aspirator (RIA) Aspirate Collected from Patients Undergoing Hip Arthroplasty. 
Journal of Orthopaedic Research, 27, 42-49. https://doi.org/10.1002/jor.20715

[13] Danckwardt-Lillieström, G., Lorenzi, L. and Olerud, S. (1970) Intracortical Circulation after Intramedullary Reaming with Reduction of Pressure in the Medullary Cavity. The Journal of Bone and Joint Surgery. American Volume, 52, 1390-1394. https://doi.org/10.2106/00004623-197052070-00013

[14] Schemitsch, E.H., Kowalski, M.J., Swiontkowski, M.F. and Senft, D. (1994) Cortical Bone Blood Flow in Reamed and Unreamed Locked Intramedullary Nailing: A Fractured Tibia Model in Sheep. Journal of Orthopaedic Trauma, 8, 373-382. https://doi.org/10.1097/00005131-199410000-00002

[15] Nakamura, T., Itoman, M. and Yokoyama, K. (1999) Cortical Revascularization after Reamed and Unreamed Intramedullary Nailing in the Rabbit Femur: A Microangiographic Histometric Analysis. The Journal of Trauma, 47, 744-751. https://doi.org/10.1097/00005373-199910000-00022

[16] Reynders, P.A. and Broos, P.L. (2000) Healing of Closed Femoral Shaft Fractures Treated with the AO Unreamed Femoral Nail. A Comparative Study with the AO Reamed Femoral Nail. Injury, 31, 367-371.

https://doi.org/10.1016/S0020-1383(00)00005-X 